\title{
In focus in HCB
}

\author{
Douglas J. Taatjes ${ }^{1}$ (D) Jürgen Roth $^{2}$
}

Accepted: 15 November 2016 / Published online: 29 November 2016

(C) Springer-Verlag Berlin Heidelberg 2016

The publications in the first issue of HCB in 2017 reflect the scope of the journal well by applying histochemical, immunohistochemical and in situ molecular techniques to problems of organ development and cell differentiation (Adhikari et al. 2017; Kuriyama et al. 2017; Nakata et al. 2017; Castro et al. 2017), as well as cellular and surgical pathology (Mühlfeld et al. 2017; Bačová et al. 2017; Limberger et al. 2017) and the development and improvement of methods useful in histochemistry and cell biology (García-Martínez et al. 2017; Couppé et al. 2017; Chawla et al. 2017).

The role of the transcription factor grainyhead-like 3 (Grhl3) for epithelial morphogenesis of the circumvallate papilla (CVP) during mouse development was studied by Adhikari et al. (2017). CVP represents an excellent model system to analyze induction, epithelial thickening and bud formation which results in a dome-shaped protrusion from an epithelial surface that in the adult converts to CVP. Previously, various signaling pathways such as Shh, Wnt11, Fgf10, Pax9, Six1 and 4, Eda and WT1 were shown to be important for the interaction between epithelium and underlying mesenchyme during CVP morphogenesis. The expression of Grhl3 as shown by in situ hybridization was restricted to the thickened epithelium (E 13.5) and the apex and trench epithelium (E 14.5, E 15.5). In an in vitro tongue culture model of E 13.5, the effect of Grhl3 knockdown on cell migration, proliferation and apoptosis was investigated.

Douglas J. Taatjes

douglas.taatjes@uvm.edu

1 Department of Pathology and Laboratory Medicine, The University of Vermont College of Medicine, Burlington, VT 05405, USA

2 University of Zurich, 8091 Zurich, Switzerland
The Grhl3 knockdown resulted in a smaller apical region of CVP and more deeply invaginated epithelial stalks. This was associated with increased cell proliferation (Ki67 immunostaining), reduced apoptosis rate (TUNEL assay) and alterations of the actin cytoskeleton. Immunostaining for ROCK1 and E-cadherin was almost absent in the apex and vallum epithelium. In the trench epithelium, ROCK1 was strongly reduced but E-cadherin was increased. By RTqPCR, expression of Shh, Ptch1, Gli3, Wnt11 and Cnnb1 was increased. It was concluded that Grhl3 has a specific role in the formation of the epithelial CVP component and of the epithelial invagination.

Uroplakins (Upk) are components of the rigid plaques of the urothelial surface epithelium and exist as four proteins, with Upk3 representing a main structural urothelium protein. Kuriyama et al. (2017) have investigated the tissue-specific expression of Upk3b in transgenic embryonic and adult mice expressing Cre recombinase driven by the $U p k 3$ promoter. In transgenic embryonic mice, Cre recombinase activity as detected by tandem dimer (td)Tomato was found in cranial and trunk neural crest cells, heart, liver, lung and kidney, as well as developing gonad cells. The analysis of adult mice focused on genital organs. In testis and epididymis, elongating spermatids and spermatozoa were positive for UPK3 and tdTomato. In the ovary, UPK3 and tdTomato were undetectable in primary follicles, but gradually increased from secondary follicles to vesicular follicles. The mucosal epithelium of the fallopian tube was also positive. Of note, in both male and female genital organs, distinct differences in the expression pattern of endogenous UPK3B (as visualized by IHC) and of Upk3b-Cre activity were observed. The observation that UPK3B is present in sperm and in follicles, as well as in the gamete-conducting systems of both sexes, provides the basis for studies to unravel its role in gamete maturation. 
Tissue microarrays (TMAs) have become popular in the surgical pathology suite as a result of their economy of scale in allowing the analysis of multiple specimen samples on a single glass slide. For TMAs to provide accurate diagnostic results, validation studies must be performed to ensure that the cell populations present on the TMA spots accurately represent those contained in the whole sample. Limberger et al. (2017) have investigated whether TMAs from subtypes of myeloproliferative neoplasms (MPN) of bone marrow can be used as a true representative of the whole sample collected. Moreover, they performed quantitative immunohistochemical analyses of the TMA samples to compare with those results obtained from whole samples. For histological evaluation, the following six parameters were compared between the TMAs and the whole tissue sections: (1) cellularity of hematopoiesis as assessed by the percentage of hematopoietic tissue per volume within the marrow spaces; (2) ratio between granulopoiesis and erythropoiesis (G/E ratio); (3) number of megakaryocytes; (4) grade of myelofibrosis according to WHO system with Gomori silver staining; (5) content of iron by Prussian blue staining; and (6) microvessel density assessed by CD34 immunohistochemistry. The results, compared using the intra-class correlation coefficient (ICC), found that in general, ICC analyses showed moderate to high correlations of TMAs with whole tissue slides. Specifically, when assessing parameters most often affected by the MPN tissues, such as cellularity of hematopoiesis, number of megakaryocytes, microvessel density and grade of myelofibrosis, good correlations were found between the two techniques. Moreover, immunohistochemical staining with NFE2 and calreticulin on TMAs was consistent with previously reported findings. Overall, their results showed good correlation between the histological parameters assessed for TMAs and whole tissue slides, though the authors caution that TMAs should be constructed in triplicate to achieve sufficient correlation.

Bone marrow transplantation (BM-Tx) following irradiation is currently used to treat certain human hematological malignancies. Moreover, similar techniques are employed in experimental animal research to assess the impact of bone marrow-derived cells on phenotypic development in genetically altered individuals. However, it is well known that body irradiation often leads to pulmonary complications. In this light, alveolar type II cells tend to be particularly susceptible to damage from radiation sources. Using mouse models, Mühlfeld et al. (2017) have now undertaken a rigorous investigation using design-based stereological methods to address the following two issues: (1) characterization of the effects produced by irradiation and BM-Tx on alveolar type II cells and their surfactant products and (2) are type II cell and surfactant alterations induced by the above-mentioned regimen more severe in surfactant protein-deficient (SP-D) mice compared to their wild-type littermates? Both light microscopic and transmission electron microscopic images were assessed for a wide variety of stereological parameters. Their results can be summarized as follows: (1) untreated SP-D mice displayed both a higher number of larger-sized type II epithelial cells and a greater degree of surfactant-storing lamellar bodies compared to their wild-type littermates and (2) irradiation followed by BM-Tx resulted in hyperplasia, hypertrophy and formation of giant lamellar bodies in both wild-type and SP-D animals (though the extent of these alterations was exacerbated in the SD-D animals). Their results underscore the critical importance of employing rigorous design-based stereological methods in order to accurately compare by quantitative methodology morphological alterations potentially induced by experimental procedures.

\section{References}

Adhikari N, Neupane S, Gwon G-J, Kim J-Y, An C-H, Lee S, Sohn W-J, Lee Y, Kim J-Y (2017) Grhl3 modulates epithelial structure formation of the circumvallate papilla during mouse development. Histochem Cell Biol. doi:10.1007/s00418-016-1487-7

Bačová BS, Vinczenzová C, Žurmanová J, Kašparová D, Knezl V, Beňová TE, Pavelka S, Soukup T, Tribulová N (2017) Altered thyroid status affects myocardial expression of connexin-43 and susceptibility of rat heart to malignant arrhythmias that can be partially normalized by red palm oil intake. Histochem Cell Biol. doi:10.1007/s00418-016-1488-6

Castro MM, Kim B, Hill E, Fialho MCG, Puga LCHP, Freitas MB, Breton S, Machado-Neves M (2017) The expression patterns of aquaporin 9, vacuolar H+-ATPase, and cytokeratin 5 in the epididymis of the common vampire bat. Histochem Cell Biol. doi:10.1007/s00418-016-1477-9

Chawla M, Verma V, Kapoor M, Kapoor S (2017) A novel application of periodic acid-Schiff (PAS) staining and fluorescence imaging for analysing tapetum and microspore development. Histochem Cell Biol. doi:10.1007/s00418-016-1481-0

Couppé C, Svensson RB, Heinemeier KM, Thomsen EW, Bayer ML, Christensen L, Kjaer M, Magnusson SP, Schjerling P (2017) Quantification of cell density in rat Achilles tendon: development and application of a new method. Histochem Cell Biol. doi:10.1007/s00418-016-1482-z

García-Martínez L, Campos F, Godoy-Guzman C, del Carmen Sanchez-Quevedo M, Garzon I, Alaminos M, Campos A, Carriel V (2017) Encapsulation of human elastic cartilage-derived chondrocytes in nanostructured fibrin-agarose hydrogels. Histochem Cell Biol. doi:10.1007/s00418-016-1485-9

Kuriyama S, Tamiya Y, Tanaka M (2017) Spatiotemporal expression of UPK3B and its promoter activity during embryogenesis and spermatogenesis. Histochem Cell Biol. doi:10.1007/ s00418-016-1486-8

Limberger KA, Bogatyreva L, Todorova R, Herde B, Hauschke D, Pahl HL, Werner M, Aumann K (2017) Tissue microarray technique is applicable to bone marrow biopsies of myeloproliferative neoplasms. Histochem Cell Biol. doi:10.1007/ s00418-016-1476-x

Mühlfeld C, Madsen J, Mackay R-M, Schneider JP, Schipke J, Lutz D, Birkelbach B, Knudsen L, Botto M, Ochs M, Clark H (2017) Effect of irradiation/bone marrow transplantation 
on alveolar epithelial type II cells is aggravated in surfactant protein D deficient mice. Histochem Cell Biol. doi:10.1007/ s00418-016-1479-7

Nakata H, Wakayama T, Asano T, Nishiuchi T, Iseki S (2017) Identification of sperm equatorial segment protein 1 in the acrosome as the primary binding target of peanut agglutinin (PNA) in the mouse testis. Histochem Cell Biol. doi:10.1007/ s00418-016-1478-8 